\title{
Erratum: Spontaneous excitation of an atom in a Kerr spacetime [Phys. Rev. D 95, 065015 (2017)]
}

\author{
G. Menezes
}

(Received 22 December 2017; published 29 January 2018)

\begin{abstract}
DOI: 10.1103/PhysRevD.97.029901
\end{abstract}
The discussion on radiative processes of static atoms in Kerr spacetime using the formalism developed by Dalibard, Dupont-Roc, and Cohen-Tannoudji as described in [1] is inaccurate in some points. Regarding the results for a static atom coupled with a scalar field prepared in the past Boulware vacuum, the contributions from vacuum fluctuations for sufficiently large $\Delta \tau$ are of the form

$$
\begin{aligned}
\left\langle\frac{d H_{A}}{d t}\right\rangle_{V F}= & \left\langle\frac{d H_{A}}{d t}\right\rangle_{V F}^{0}-\pi \lambda^{2} \sum_{l, m} \sum_{\nu^{\prime}} \mathcal{M}\left(\nu \rightarrow \nu^{\prime}\right) \widetilde{\Delta \nu} \theta(\widetilde{\Delta \nu}) \theta\left(-\widetilde{\Delta \nu}-m \Omega_{H}\right) \mathcal{P}_{-\widetilde{\Delta \nu l m}}^{-}(r, \theta) \\
& +\pi \lambda^{2} \sum_{l, m} \sum_{\nu^{\prime}} \mathcal{M}\left(\nu \rightarrow \nu^{\prime}\right)|\widetilde{\Delta \nu}| \theta(-\widetilde{\Delta \nu}) \theta\left(-|\widetilde{\Delta \nu}|-m \Omega_{H}\right) \mathcal{P}_{-|\widetilde{\Delta \nu}| l m}^{-}(r, \theta),
\end{aligned}
$$

where the first term is the original one given in Eq. (25) of the aforementioned paper. The remaining contributions in the above equation are missing in the investigation (all nomenclature and suitable definitions of all physical quantities are properly addressed in the paper). Likewise, the contributions from radiation reaction (which are the same for all four vacuum states studied) for sufficiently large $\Delta \tau$ experience the same problem (again, for static atoms):

$$
\begin{aligned}
\left\langle\frac{d H_{A}}{d t}\right\rangle_{R R}= & \left\langle\frac{d H_{A}}{d t}\right\rangle_{R R}^{0}+\pi \lambda^{2} \sum_{l, m} \sum_{\nu^{\prime}} \mathcal{M}\left(\nu \rightarrow \nu^{\prime}\right) \widetilde{\Delta \nu} \theta(\widetilde{\Delta \nu}) \theta\left(-\widetilde{\Delta \nu}-m \Omega_{H}\right) \mathcal{P}_{-\widetilde{\Delta \nu l m}}^{-}(r, \theta) \\
& +\pi \lambda^{2} \sum_{l, m} \sum_{\nu^{\prime}} \mathcal{M}\left(\nu \rightarrow \nu^{\prime}\right)|\widetilde{\Delta \nu}| \theta(-\widetilde{\Delta \nu}) \theta\left(-|\widetilde{\Delta \nu}|-m \Omega_{H}\right) \mathcal{P}_{-|\widetilde{\Delta \nu}| l m}^{-}(r, \theta),
\end{aligned}
$$

where again the first term is the original one given in Eq. (30) of [1]. As a consequence of these new results, the total rate for large $\Delta \tau$ in the case of static atoms coupled with scalar fields prepared in the past Boulware vacuum is given by

$$
\left\langle\frac{d H_{A}}{d t}\right\rangle_{\text {tot }}=\left\langle\frac{d H_{A}}{d t}\right\rangle_{\text {tot }}^{0}+2 \pi \lambda^{2} \sum_{l, m} \sum_{\nu^{\prime}} \mathcal{M}\left(\nu \rightarrow \nu^{\prime}\right)|\widetilde{\Delta \nu}| \theta(-\widetilde{\Delta \nu}) \theta\left(-|\widetilde{\Delta \nu}|-m \Omega_{H}\right) \mathcal{P}_{-|\widetilde{\Delta \nu}| l m}^{-}(r, \theta),
$$

where the first term is the one discussed in [1]. The interpretation of such results is straightforward: for an atom prepared in the ground state, there is a fine-tuning between vacuum fluctuations and radiation reaction which prevents the occurrence of spontaneous excitation to higher levels only at $\mathcal{J}^{-}$. In turn, at $\mathcal{J}^{+}$, vacuum fluctuations and radiation reaction concur with equal portions to the spontaneous excitation of an atom in the ground state. This is due to the fact that the past Boulware vacuum state does not agree with the concept of a state which is most empty at infinity, in contrast with the Boulware vacuum state defined in Schwarzschild spacetime. This discussion complements the results presented in [1].

Concerning the other vacuum states for the case of a static atom (Unruh, Candelas-Chrzanowski-Howard, and FrolovThorne), they all have the same shift in their associated rates for $\Delta \tau \rightarrow \infty$. For the vacuum-fluctuation contribution, one finds

$$
\begin{aligned}
\left\langle\frac{d H_{A}}{d t}\right\rangle_{V F}= & \left\langle\frac{d H_{A}}{d t}\right\rangle_{V F}^{0}-\pi \lambda^{2} \sum_{l, m} \sum_{\nu^{\prime}} \mathcal{M}\left(\nu \rightarrow \nu^{\prime}\right) \widetilde{\Delta \nu} \theta(\widetilde{\Delta \nu}) \theta\left(-\widetilde{\Delta \nu}-m \Omega_{H}\right) \mathcal{P}_{-\widetilde{\Delta \nu l m}}^{-}(r, \theta)\left[1+\frac{2}{\exp \left(\frac{2 \pi\left(-\widetilde{\Delta \nu}-m \Omega_{H}\right)}{\kappa_{+}}\right)-1}\right] \\
& +\pi \lambda^{2} \sum_{l, m} \sum_{\nu^{\prime}} \mathcal{M}\left(\nu \rightarrow \nu^{\prime}\right)|\widetilde{\Delta \nu}| \theta(-\widetilde{\Delta \nu}) \theta\left(-|\widetilde{\Delta \nu}|-m \Omega_{H}\right) \mathcal{P}_{-|\widetilde{\Delta \nu}| l m}^{-}(r, \theta)\left[1+\frac{2}{\exp \left(\frac{2 \pi\left(-|\widetilde{\Delta \nu}|-m \Omega_{H}\right)}{\kappa_{+}}\right)-1}\right] .
\end{aligned}
$$


The first term in the right-hand side of the above expression (the one with the superscript 0 ) describes the contributions which were already present in [1], namely, Eq. (36) for the Unruh vacuum state, Eq. (43) for the Candelas-ChrzanowskiHoward vacuum state, and Eq. (49) for the Frolov-Thorne vacuum state. By taking into account the shift in the radiationreaction terms previously described, one can easily calculate the related shift in the total rates. In any case, it is easy to see that such shifts do not alter qualitatively the conclusions that were already drawn concerning such vacuum states; in particular, when the atom is at the region $\mathcal{J}^{-}$, the previous conclusions remain the same. Nevertheless, we like to recast a specific assertion concerning the Unruh vacuum state (again, in the case of static atoms), a topic which is unrelated to the issues raised above. In [1], it is stated that "there is thermal radiation from the rotating black hole only for $|\widetilde{\Delta \nu}|>m \Omega_{H}$ "; otherwise, "the atoms in the ground state do not get excited." From Eq. (39) in the paper, it is easy to see that this would be partially true only for a fixed $m>0$. In particular, the $m=0$ contribution ensures the existence of thermal radiation from the black hole in any given scenario, which is the expected conclusion.

We remark that the calculations concerning the rate of variation of energy for a stationary atom with zero angular momentum are accurate and, as such, the associated results are entirely trustworthy.

In summary, such adjustments not only make the conclusions of [1] more sound but they also reconcile the results for the Boulware vacuum regarding both states of motion of the atom (static and stationary with zero angular momentum). Indeed, since the past Boulware vacuum contains at future null infinity an outward flux of particles due to the Unruh-Starobinskii effect, one should expect this phenomenon to emerge in both situations. Such rectified conclusions will be notably important in the entanglement dynamics of two 2-level atoms in a Kerr spacetime [2].

[1] G. Menezes, Spontaneous excitation of an atom in a Kerr spacetime, Phys. Rev. D 95, 065015 (2017).

[2] G. Menezes, Entanglement dynamics in a Kerr spacetime, arXiv:1712.07151. 